Viewpoint

\title{
The Invention of an Optical Antenna-A Personal Reminiscence
}

\author{
Wolfgang Dieter Pohl
}

Wolfgang Dieter Pohl c/o Department of Physics, University of Basel, Klingelbergstrasse 82, 4056 Basel, Switzerland; dieter.w.pohl@gmail.com

Received: 26 October 2018; Accepted: 13 November 2018; Published: 22 November 2018

check for updates

\begin{abstract}
The subject of this article is my personal path from the near-field optical (NFO) microscope to the optical antenna and its potential for modern optics.
\end{abstract}

Keywords: breaking the diffraction limit; optical antenna; nonlinearities; antenna resonance; lifetime of Q-dots

One day in 1998, when walking through my city, I watched the TV antennas on the roofs of the houses. Most of them were dipole-shaped and equipped with a series of directors and reflectors. I began to contemplate ... How was the radiation converted into a measurable alternating voltage at the narrow gap in the center?

In my imagination, I saw how the electrons were swapping back and forth in the antenna rods, how the gap hindered their motion and how, in response, a large electric field built up in the gap. This gap was arbitrarily small compared to the length of the antenna. Since the latter is roughly half a wavelength, the field in the gap is a typical, no: the archetypical nearfield!

Being an optics man and not an electrical engineer, this was quite an exciting finding. After invention [1] of the near-field optical (NFO) microscope, the first microscope that broke the diffraction limit [2], I had been searching for the optimum shape of a NFO probe. Both types that we used-small aperture and tip—had certain drawbacks.

But now I learned that electro-magnetic technology had solved this problem for a long time already - though for wavelengths roughly 1 million times longer than that of optical waves. To illustrate that, imagine an antenna for a wave not one million times shorter but larger than the TV antenna: It would extend roughly $700 \mathrm{~km}$ from one end to the other-the distance between Boston and Washington with a $\sim 30 \mathrm{~km}$ wide gap in the New York area. Such an antenna, by the way, would resonate near AC frequency.

In 1999 I had to give a talk at the Second Asia-Pacific Workshop on Near Field Optics in Beijing. I decided to speak about the antenna concept [3]. It actually was a stopgap since I had no other really novel result at that time. To my surprise, the audience was quite intrigued by the idea. This encouraged me to further study technical antennas and, being an experimentalist, to try to build an "optical antenna".

At Basel university, our NFO team consisted of Bert Hecht, Hans Eisler, myself, and the two PhD students Peter Mühlschlegel and Javad Farahani. The optical dipole antennas required lithographic tools with $\sim 10 \mathrm{~nm}$ precision for fabrication. Such instruments (generally) became available around the turn of the century. We produced our antennas at the Eidgenoessische Materialpruefungsanstalt (EMPA) [4] with a newly acquired FIB microscope. Our test patterns (gold on glass) consisted of dipoles of 100-400 $\mathrm{nm}$ length in different orientations and, for comparison, of non-split rods of the same length as the dipoles. 
The next problem concerned the method of detection. The expectation was that a large intensity would build up in the gap between the antenna arms and not on the full length of the antenna. But how to demonstrate this? Direct imaging by classical microscopy would provide a resolution of $\lambda / 2$. That was the full length of the antenna. A near-field microscope, on the other hand, would have the necessary resolution but heavily disturb the image by its very presence ...

The 'focusing' action of the antennas hence could only be proven indirectly. I had the strange idea to look after nonlinear effects for this purpose-strange because nonlinear effects such as frequency doubling and higher order nonlinearities are weak. It was quite improbable that the nanometer-size gap volume could produce a detectable signal.

Following my inner voice I still insisted on looking for signs of nonlinearity. This was easy enough: Our standard setup consisted of a pulsed IR laser and a confocal microscope which imaged the substrate with the dipoles and rods in reflection. PhD student Peter who ran the experiment simply had to place an additional notch filter into the reflected beam. The incident IR radiation was strongly suppressed now but-lo and behold—-there were spots of IR and visible light at the position of some antennas!

Further experiments revealed various blue-shifted components which increased with second, third and even fourth power of the incident intensity—a clear indication of nonlinearity. Moreover, this radiation was visible only from properly oriented antennas with gap, having a certain length, and not on equal-length rods [5]. This confirmed the expectation of a nanometer-size, intense light spot at the gap, the center of the antenna.

The view of the light spot was completed by a simultaneous experiment of our colleagues Hans and Javad. They scanned an optical dipole over a film that contained single, fluorescing Q-dots. When the center of the antenna came close to the Q-dot a large reduction of lifetime and an increase of intensity were observed [6]. Such a behavior is typical for fluorescence in a strong field.

The end of these experiments marked also the end of our team at Basel University. Four of us were contracted by other universities or industry; I had reached the age limit and retired, heavy-heartedly in view of all the questions and opportunities that became apparent in the course of the antenna experiments.

The phenomena as well as their selectivity regarding direction, polarization, and resonance frequency bore - and, to my opinion, still bear-potential. Their derivation from electrical engineering has caused a new view of some optical phenomena. They link into various fields like quantum optics and electronics, communication, manipulation and, last not least, microscopy.

Electrons in a metal feel their finite mass at optical frequencies. The plasmon dispersion curve-indistinguishable from the photon curve at low frequencies-bends towards the horizontal. It saturates at the plasma frequency $\omega p$. In the transition region the residual conductance may still allow for some electro-technical applications, for instance rectification or multiplexing of antenna currents.

Beyond this range $(\omega>\omega p)$, however, all metals become insulators. That pulls the carpet from under conventional electric-magnetic circuitry which depends on long paths of conduction. The optical/uv regime is the end of the long-pursued engineering trend towards higher frequencies, and the optical antenna may be one of the last devices designed to the rules of conventional electromagnetic wisdom.

Acknowledgments: Thanks go to my collaborators-too many over the years to be cited individually (Consult my publication list [7] for names) - and to the colleagues and friends of the outstanding "NFO" community. Without them, this work would have been just another episode in the ever-lasting struggle for progress in science.

Conflicts of Interest: The author declares no conflicts of interest

\section{References and Notes}

1. Pohl, D.W. Optical Near-Field Scanning Microscope. European Patent Application No. 0112401, 27 December 1982. U.S. Patent 4,604,520, 20 December 1983.

2. Pohl, D.W.; Denk, W.; Lanz, M. Optical stethoscopy: Image recording with resolution $\lambda / 20$. Appl. Phys. Lett. 1984, 44, 651-653. [CrossRef] 
3. Pohl, D.W. Near field optics seen as an antenna problem. In Near-Field Optics: Principles and Applications, Proceedings of the The Second Asia-Pacific Workshop on Near Field Optics, Beijing, China, 20-23 October 1999; Ohtsu, M., Zhu, X., Eds.; World Scientific: Singapore, 2002; pp. 9-21, ISBN 981-02-4365-0.

4. Special thanks go to Ph. Gasser, Eidgenoessische Materialpruefungsanstalt (EMPA) in Dübendorf, Switzerland.

5. Muehlschlegel, P.; Eisler, H.-J.; Martin, O.J.F.; Hecht, B.; Pohl, D.W. Resonant Optical Antennas. Science 2005, 308, 1607. [CrossRef] [PubMed]

6. Farahani, J.N.; Pohl, D.W.; Eisler, H.-J.; Hecht, B. Single Quantum Dot Coupled to a Scanning Optical Antenna: A Tunable Superemitter. Phys. Rev. Lett. 2005, 95, 017402. [CrossRef] [PubMed]

7. Pohl, D.W. Publications and Patents. Available online: https://www.physik.unibas.ch/fileadmin/user_ upload/physik-unibas-ch/06_People/06_Emeriti/PohlDieter_2017_Complete_list_of_Publications.pdf (accessed on 14 April 2014).

2018 by the author. Licensee MDPI, Basel, Switzerland. This article is an open access article distributed under the terms and conditions of the Creative Commons Attribution (CC BY) license (http://creativecommons.org/licenses/by/4.0/). 